\title{
AoIP / AVB Technology and Application of Digital Audio Network*
}

\author{
Yi Guo ${ }^{1}$ and Qiong $\mathrm{Li}^{2}$ \\ 1 School of Electrical Engineering and Electronic Information, Xihua University, \\ Chengdu,China.lpngy@qq.com \\ ${ }^{2}$ China Electronics Technology Group Corporation No.52 Research Institute, \\ Hangzhou,China.1046294042@qq.com
}

\begin{abstract}
With the rapid development of Ethernet and IP technology, digital audio broadcasting over IP becomes the urgent question for radio stations. This paper analyses the facing problems for real-time transmission of digital audio over IP network, and discusses the corresponding solutions, focusing on the introduction of AoIP and AVB technology, and gives the application scheme of the actual. The solution also has a large number of applications in actual projects, which has the reality instruction significance.
\end{abstract}

Keywords: Digital Audio Broadcast; QoS; CobraNet; AoIP; AVB

\section{Introduction}

In broadcast and television professional occasions ,the transmission of real-time digital audio is usually by AES3 (AES / EBU) or AES10 (MADI), with the rapid development of Ethernet (Ethernet) and IP technology, people have been looking forward to take advantage of Ethernet and IP network transmission to achieve this task, Because Ethernet is the most widely used and most cost-effective digital network, Because of our collection and transmission of data information, monitoring information has become increasingly dependent on IP networks [1-3]. So if the real-time audio transmission can be compatible with existing Ethernet, System architecture will become simplified, Room wiring will also become simpler and while enhancing Ethernet switching features, also help to change the traditional digital audio's limitations which only rely on point-to-point transmission, to achieve digital audio transmission soft switch and soft route [4-5].

The emergence of CobraNet locally to solve this problem, although it uses the same cables and switching equipment. Because of its incompatibility with the IP protocol must lead us to establish a closed network for it alone, while its transmission capacity, delay and so on, which have also been greatly restricted. Until the appearance of AoIP and AVB, This problem will be completely resolved, People's doubts to Lossless of IP network traffic in real-time audio will be eliminated, AES Formally issued the first use of the existing IP transport networks in real-time streaming of high-quality audio universal standard AES67- 2013 in September last year, AoIP (Audio over IP) technology to become part of the professional audio; On the other hand, AVB standards are in full swing, this standard is leaded by the Institute of Electrical and Electronics Engineers (IEEE),by expanding the existing IEEE 802 standard, will solve the bottleneck of Ethernet-based audio and video transmission ,to achieve real-time audio and video flows Ethernet bridging, transport and switching.

A new multimedia network applications era is coming, it will deeply change the radio, television, professional audio, security, automotive electronics and other fields, Some people describe it as much as Film cameras into the digital camera, the traditional audio

\footnotetext{
* This article is supported by ChunHui project of the Ministry of Education (Grant No: z2014054).

This article is supported by the Key scientific research fund of Xihua University (Grant No: z1320927).
} 
and video design is being challenged, and simple and efficient, more powerful new system will continue to emerge, This paper attempts to presentations and discussions AoIP and AVB technical, from a development perspective, to look forward to their future broadcast audio production and broadcasting system brought opportunities and driving force, so that we have a good mental preparation and knowledge to welcome come to this era.

\section{AoIP / AVB Technical Analysis}

\subsection{Ethernet / IP Network Several Issues Need to be Solved in Real-Time Audio Transmission}

In the beginning, Ethernet (Ethernet) is used to connect computer, and it follows the IEEE802.3 standard, using a logical bus topology structure and CSMA / CD Carrier Sense collision detection technology to exchange information, which is proof Ethernet inherently cannot use to transmit real-time audio and video.

Located in the higher-level TCP / IP protocol, usually equipped with Ethernet as a carrier in LAN, but because of its roots is the Internet protocol, delays are not the same when IP packets through different routes, lead to it to reach the end of the sequence and delay uncertainty ; RTP (Real-time Transport Protocol Real-time Transport protocol) to some extent, to solve this problem and enhance the flow of stability, but it cannot transmit synchronous clock, only to be used for network-on-demand and other non-professional way.

Transfer the media clock is the first problem needed to solve in Ethernet / IP network in real-time audio transmission, Figure 1 illustrates this process:

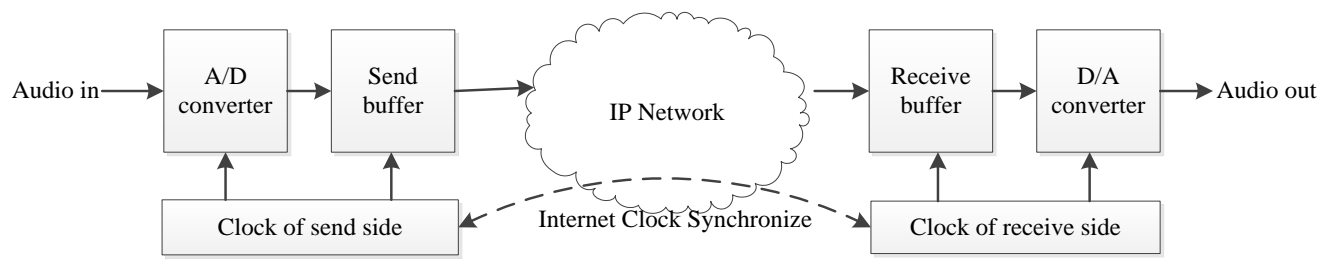

Figure 1. Ethernet / IP Network Transmission Medium Clock Schematic

Audio data after A / D conversion with its own sampling clock (same applies to AES3 input), which we usually call it as the $48 \mathrm{~K}$ sampling clock, audio, this $48 \mathrm{~K}$ come from internal crystal oscillator in the sender device, the digitized audio data by the cache to pack into data packets send to receiving end, and then by recovering the audio data after D / A conversion, However, D / A clock comes from the crystal oscillator in receiving end device, while the same nominal $48 \mathrm{~K}$, in fact, is not synchronized with the sender of the two clocks, two unsynchronized clocks will cause buffer sometimes overflow or underflow, it is called "sonic boom" in sound quality, in order to reduce the "sonic boom" appears, can buffer resampling which is also called that sample rate conversion method, the larger buffer, the better the sound quality, but with the increase of buffer ,increased transmission delay will be followed, The output of audio data is not the original data, which cannot be achieved lossless transmission, At present, some manufacturers of IP listener is actually used such programs, but if used as a high-quality transport services, such as generation of broadcasting, it is insufficient.

If you can use the network to synchronous the sender and the receiver clock, the problem will be solved, such as generating a standard reference clock in the network (network clock), which is used to synchronize the sampling clock(media clock) of each node, the network does not exist data overflow problem, IEEE1588 provides this possibility, IEEE1588 stands for "network measurement precision clock synchronization 
protocol and control systems standards", the master clock with the network to exchange information packet contains a timestamp periodically, After receiving the clock, the clock offset measurement and delay measurements using an offset to correct the local clock, this program can make use of the existing IP network without any changes, but the audio equipment to support the conversion from internal IEEE1588 clock; In AVB system using another precise time synchronization protocol (PTP) - IEEE 802.1AS, as a simplified version of the IEEE 1588 protocol, IEEE 802.1AS and 1588 the biggest difference is that the PTP is based entirely on two layer network, non-IP routing protocols, in maximum 7hop network environment, in theory, be able to guarantee the PTP clock synchronization error within $1 \mu$ s.

Complete Ethernet / IP network transmission of real-time audio lossless and it second condition is bandwidth reservation, or network quality of service (QoS).

There are a lot of cars and pedestrians on the road, corresponding to the different services in the network packets, the road is often Occur congestion situation, the network is no exception, which we really doubt whether the audio with other data transportation will not be crowded together and delay reasons, the solution is to divide the packets with different priorities, by different quality of service provided to achieve, like the road divided into different exclusive lanes, the same priority clearance for emergency vehicles. Use QoS classification criteria of DSCP (Differentiated Services Code Point), each of the reference clock data header is set as the highest priority; after that audio data belonging to the second high priority; control signals and other signals in a lower priority level. As a result, to ensure smooth flow and low jitter of audio stream signal. Today has been widely used for Gigabit Ethernet bandwidth, and the bandwidth in the idle state in most cases, with QoS control, real-time audio transfer is not a problem.

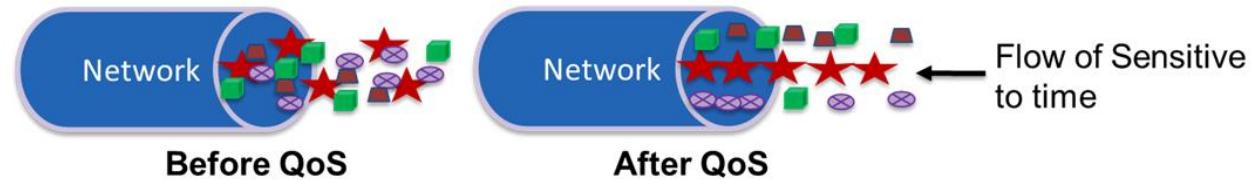

Figure 2. Network QoS Schematic

AVB will also use the IEEE 802.1Qat stream reservation protocol and IEEE 802.1Qav queue and forwarding protocol for time-sensitive audio and video signals for resource reservation, and to ensure that the traditional asynchronous Ethernet data traffic does not interfere with the real-time audio AVB video stream.

These two problems are resolved, the remaining issues as well as the transport layer, coding layer, control layer, next will introduction about it with specific programs and protocols.

\subsection{AoIP Technical Standards and AES67-2013}

AoIP (Audio over IP), which uses IP network to transfer high fidelity audio, the audio in here refers to at least $44.1 \mathrm{~K}$ sampling, quantization 16 more than or equal to CDquality audio. AoIP has different with VoIP (Voice over IP), VOIP is used for voice communication, transmission of telephone-quality low bit rate signals.

The second feature of AoIP is use of the existing IP network, and delay is less than 10 milliseconds .a decade ago, Axia Audio (Telos branch network audio) introduced the Livewire, which use for uncompressed digital audio recording, then there appears Dante, RAVENNA, Q-LAN, WheatNetIP etc. These AoIP technology from different manufacturers, the common denominator is (1) almost all using IEEE1588 as synchronization resource of media clock (2) use of existing IP protocol, such as the transport layer uses UDP / RTP, QoS using DiffServ, etc., Table 1 from AES technology trends report in 2011: 
Table 1. Current Major AoIP Technology

\begin{tabular}{|c|c|c|c|c|}
\hline Technology & $\begin{array}{c}\text { development } \\
\text { company }\end{array}$ & launch date & $\begin{array}{c}\text { synchronization } \\
\text { scheme }\end{array}$ & $\begin{array}{c}\text { transport } \\
\text { protocols }\end{array}$ \\
\hline LiveWire & Telos/Axia & 2004 & $\begin{array}{c}\text { IEEE1588 } \\
\text { Developing })\end{array}$ & RTP \\
\hline Dante & Audinate & 2006 & IEEE1588-2002 & UDP \\
\hline Q-LAN & $\begin{array}{c}\text { QSC Audio } \\
\text { Products }\end{array}$ & 2009 & IEEE1588-2002 & UDP \\
\hline RAVENNA & ALC Network & Developing & IEEE1588-2002 & RTP \\
\hline
\end{tabular}

AES code for standardization working group SC-02-12-H, and in December 2010 launched a project called the X192, its purpose is not to invent new technology, but define an interoperable program on the basis of prior art, which achieve interoperability between equipment from different vendors AoIP, standard formally promulgated in September 2013, named AES67-2013 [6].

AES67-2013's full name is "AES standard for audio applications of networks-Highperformance streaming audio-over-IP interoperability ",translated into Chinese is "interoperable AoIP fidelity audio streaming application standards", the standard is divided into 10 parts, including provisions media clock synchronization, coding, transmission, and connection management, in addition, the standard explained on the compatibility with the AVB .

So far, ALC NetworX RAVENNA has announced its support AES67, and Audinate will to firmware upgrade program, which will have to upgrade to AES67 products in the second half of 2014.

\subsection{AVB Technology Introduced}

AVB full name is Ethernet audio video bridging (Ethernet Audio / Video Bridging), and it is a new IEEE 802 standard. AVB from a 802.3 research team, and IEEE 802.1AVB working group was established in November 2005 ,began to develop a series of agreements, to enhance existing 802 network functions, making Ethernet-based realtime audio and video transmission technology gradually from planning phase to experimental phase, and ultimately to the market.

AVB has different with AoIP technology, AVB needs to improve the communication protocol of second floor, to solve problem in the transmission of audio and video data stream, greatly improve the transmission control ability .In addition to meeting audio transmission, supports 256 different formats of audio and video data streams (including the sampling frequency) to transfer in the same network, without any interference, and transmission delay compressed into microseconds.

In order to provide real-time streaming media services, which is synchronization and low latency, needing to establish a AVB network, called AVB "cloud". As shown in Figure 3 ,AVB "cloud" requires full-duplex Ethernet link, at least over the speed at $100 \mathrm{Mbps}$, and equipped with AVB and AVB switch terminal equipment, logical link discovery protocol (IEEE 802.1AB - LLDP). 


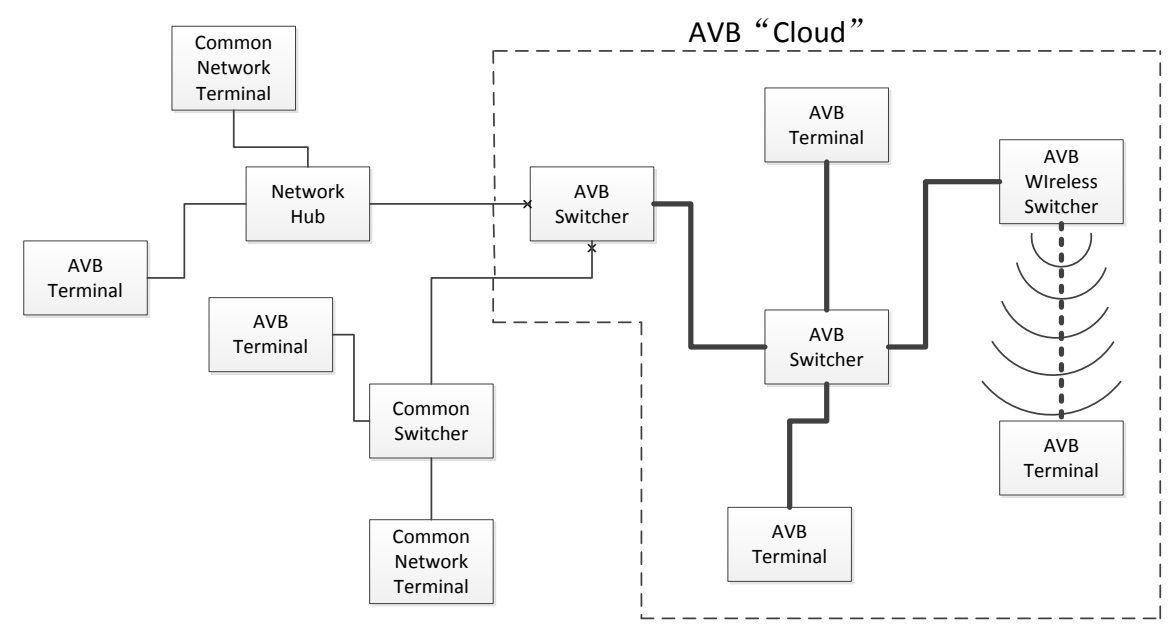

Figure 3. AVB Cloud Schematic

Within AVB "cloud", due to the delay and quality of service are guaranteed, can provide real-time high quality streaming media services. Meanwhile, AVB network remains compatible with the conventional Ethernet, it is possible to connect a traditional switches, hubs, and the terminal device. However, due to the half-duplex nature of the hub, as well as traditional Ethernet switches do not have AVB function, cannot fully guarantee its streaming real-time services, so out of AVB "cloud", only to protect the ordinary maximum delivery capabilities and connected AVB network.

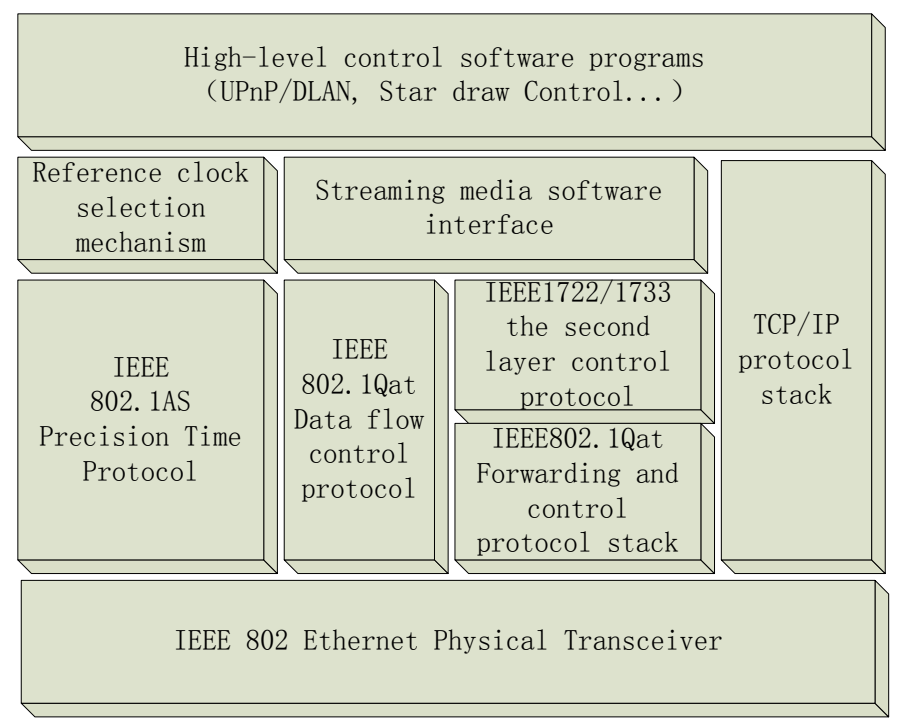

Figure 4. AVB Protocol Set Level in the OSI Model

Figure 4 reflects the internal structure of the AVB standard system, we can see that, AVB across multiple levels of data link layer and the network layer, involving most of the levels of TCP / IP protocol suite, but its structure is essential to maintain and compatible with existing Ethernet system, just extend some features, increase and revised agreement include:

(1) Precise time synchronization protocol --IEEE 802.1AS

(2) Stream reservation protocol - IEEE 802.1Qat

(3) The queue and forward protocol - IEEE 802.1Qav

(4) Audio Video Bridging Systems - IEEE 802.1BA 
In addition, there are two other using IEEE 802.1 AVB to provide high-quality professional audio and video transmission:

(1) Audio Video Bridging Transfer Protocol (second floor) --IEEE 1722

(2) Real-time Transport Protocol (three) - IEEE 1733

The end of AVB standard system formulating, and most of them have been officially promulgated standards; August 31, 2009 - an ad hoc industry organization --AVnu Alliance coalition, there are two driving forces in the development of Ethernet AVB, the first group is represented by Harman and BMW system vendors, another group is Broadcom, Marvell and Xilinx chip manufacturers, With the development of AVB commercial, whether professional or civilian, AV industry is entering a new era of digital network.

\subsection{About Dante}

We introduced AoIP and AVB, it should be said they are not contradictory. As a special case of audio, AOIP should be inclusive in AVB in the future, whereas equipment and mechanisms of AVB gave AoIP greater capacity and better stability.

Involved in a road map problem here, in fact, the current AoIP technology is a mature and inspection technology with a lot of time and examples, as can be seen from Table 1, the mainstream vendors have the promotion period over five years, and have large number of optional products. Now only involves an agreement to consistency,

AoIP and AVB one difference is that AoIP can be run for any existing local area network without modification, but AVB must separately purchase and reconstruction AVB switch network, and is currently AVB technology is still in development. If we choose a technology to achieve AoIP, and in accordance with standard AES67-2013, become as AVB system in the future, would be a good solution.

Here we recommend Dante, Dante from Audinate companies in Australia, is the most mature, the highest market share of products,

there are more than 100 companies choose Dante audio network audio transmission as standard, including Harman, NTP, YAMAHA, DHD, Soundcraft professional audio equipment and other leading companies, including the large number of successful applications in the 2012 London Olympics; The difference with RAVENNA, Dante is not only written the protocol specification in the documentation, it has a standard hardware and software solutions and complete device management and routing management mechanism, Dante equipment from different vendors with good compatibility and connectivity features, you can be managed on the same platform in network ,in addition, Dante is AVnu's main sponsor through firmware and software upgrades with the form of the White Paper commitment, and seamless support AVB standard (currently its hardware devices already have AVB condition).

Dante main features include:

(1) More channels and lower latency, the number of measured through Gigabit Ethernet (48K / 24bit) over 400, a single-hop delay shortest 83.3 microseconds

(2) Using Zeroconf protocol automatically find and configure the device, eliminates the need for users to manually set the IP process

(3) Supports unicast and multicast, can effectively optimize network traffic

(4) While providing a standby two redundant channels

(5) Provides PC and MAC-side virtual sound card, the card directly from this site transmit real-time audio stream

(6) All configurations are stored locally on the electrical signal is routed directly after opening 


\section{Aoip / AVB Compare with Cobranet and Aoip / AVB's Advantages}

\subsection{The Introduction of CobraNet}

The late nineties, the US PeakAudio company launched the Ethernet audio transmission scheme called --CobraNet glasses network, the company was acquired chip maker CIRRUS LOGIC, and to provide supporting card and special chip methods to get CobraNet rapid promotion [7].

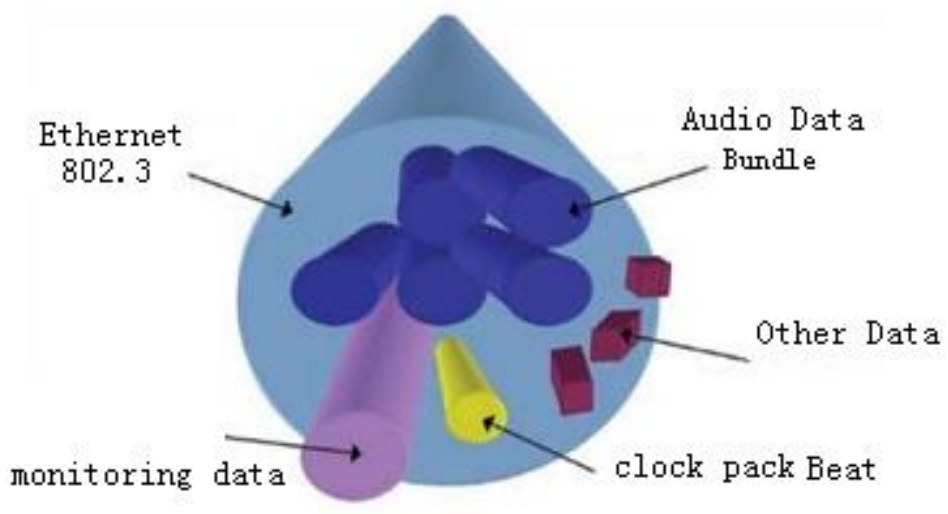

Figure 5. Internal Communications of Cobranet

CobraNet obtains the sample clock synchronization network with a device (Conductor) continues to send packets beat (Beat Packet), although CobraNet is a synchronous network, but it does not use a standard such as IEEE1588 or IEEE802.1; The Audio transmission of CobraNet is achieved by Bundle, Bundle is CobraNet unique package, each Bundle contains eight 20-channel audio data, each CobraNet device up to receive 8 Bundle, and supports multi - Unicast Bundle.

CIRRUS LOGIC provides CM-2 solution is limited, Single CobraNet device support maximum conversion channel number 16 and 16, In the one-way 100M Ethernet can transmit $6448 \mathrm{kHz}$, 20bit audio signal path, CobraNet codec delay is fixed, there is $1.33 \mathrm{~ms}, 2.66 \mathrm{~ms}$ and $5.33 \mathrm{~ms}$ three kinds to choose from. For stability considerations, factory recommended $5.33 \mathrm{~ms}$; In addition to audio signals, CobraNet can also transfer RS485 serial communication data and other non-IP data synchronization, device management support SNMP.

Audio transmission network solutions of professional audio field as well as France Digigram's EtherSound, due EtherSound little impact on the market, and the company has now changed Digigram push AoIP program, will not be introduced here.

\subsection{CobraNet Defects and AoIP / AVB Advantage}

From the point of historical development, CobraNet Ethernet Audio made a great contribution, even now CobraNet is a relatively mature engineering program, but with the advent of AoIP and AVB technology, its flaws have gradually surfaced, many advantages also being lost.

\subsubsection{AoIP / AVB Standard with Greater than CobraNet}

Although CobraNet built on the basis of standard Ethernet, but it's synchronization mechanism, the transmission mechanism is unique, is a non-public enterprise standards, whether beat packet or data Bundle packages are directly encapsulated into a MAC frame two-story, regardless of the IP, but did not use UDP, RTP and other transport protocol. It is not counted as AoIP, although each CobraNet device at boot time will get a dynamic IP 
address, but the IP address is not a service for CobraNet itself, but the use of other nonsynchronous senior management software information.

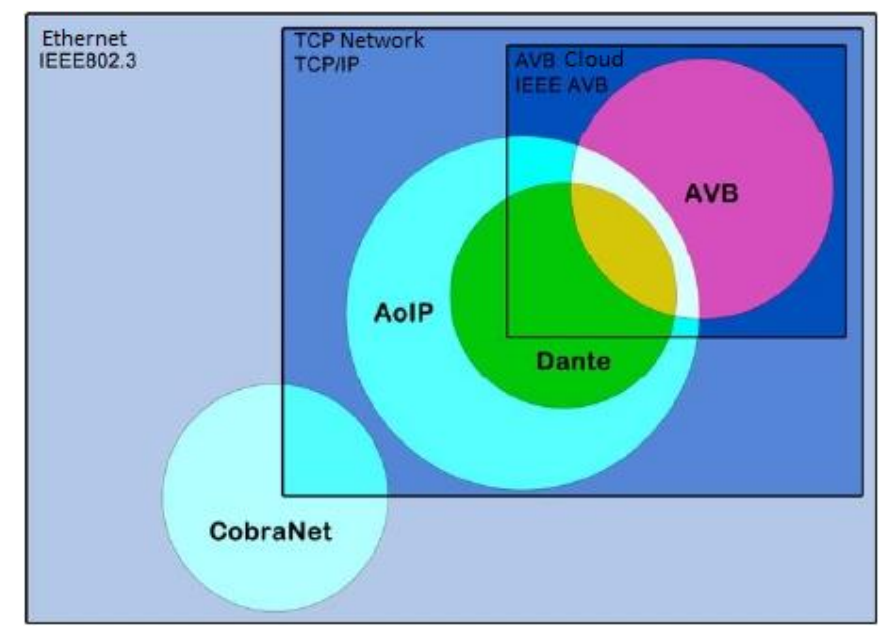

Figure 6. Major Network Protocols and Network Audio Platform
Relationship Diagram

Figure 6 shows the relationship between the main network audio protocol and network platform, you can see CobraNet Ethernet is a special application located on the second floor, has no intersection with AoIP and AVB, that it cannot support the Association AES67-2013 and AVB, so CobraNet is not the future direction of the real-time transmission of audio technology, along with new technologies to produce and mature stage of history will slowly fade.

\subsubsection{AoIP / AVB has Greater Compatibility and Flexibility than CobraNet}

CobraNet cannot use QoS to allocate their bandwidth and priority conflicts and thirdparty IP data bandwidth problem is inevitable, So when you need coexisted with other IPbased business, CobraNet will become unstable, the only effective way is to build a separate network for CobraNet, and then give complete management to CobraNet ,In practical engineering always doing it, of course CobraNet via SNMP or serial bridge to transfer some control signals, but the performance is very low. For the most part, only to build a new IP network for the exchange of other data, if you consider each redundancy, you need to open 4 network, which is obviously very uneconomical, Figure 7 illustrates this point.
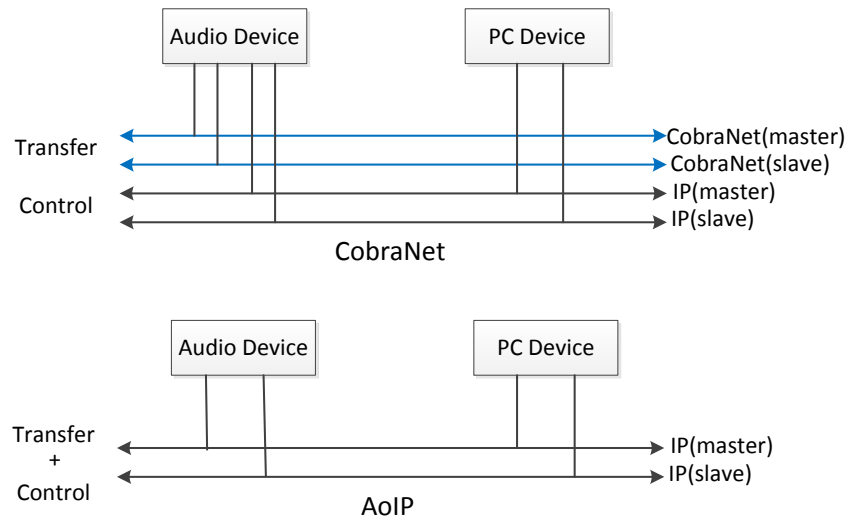

Figure 7. The Different Structures of CobraNet and AoIP under Mode Network 
In addition, CobraNet equipment interconnection between different vendors is not easy need depth configuration of VLAN and Bundle number with Professionals, exhibit poor compatibility. Table 2 lists the main characteristics and CobraNet AoIP comparison:

Table 2. AolP with CobraNet Comparison Table

\begin{tabular}{|c|c|c|}
\hline Comparison item & CobraNet & AoIP（take Dante for instance） \\
\hline Fully support Ethernet & Yes & Yes \\
\hline Fully support TCP/IP & No & Yes \\
\hline Support AES67-2013 & No & Yes \\
\hline Sampling Rate & $48 \mathrm{~K} / 96 \mathrm{~K}$ & $44.1 \mathrm{~K} / 48 \mathrm{~K} / 88.2 \mathrm{~K} / 96 \mathrm{~K} / 192 \mathrm{~K}$ \\
\hline Quantification Bits & $\begin{array}{l}\text { 16/20/24bit (20bit } \\
\text { recommend) }\end{array}$ & 16/24/32bit (24bit recommend) \\
\hline $\begin{array}{l}\text { Transport Channel Number } \\
\text { for Each } 100 \mathrm{MB}\end{array}$ & $\begin{array}{c}64 \text { Entries (20bit) }, 56 \\
\text { Entries (24bit) }\end{array}$ & 56 Entries（24bit） \\
\hline $\begin{array}{l}\text { The Actual Delay for Each } \\
100 \mathrm{MB}\end{array}$ & $5.33 \mathrm{mS}$ & $150 \mathrm{uS}$ \\
\hline Unicast and Multicast & limited supporting & fully support \\
\hline QoS & $\begin{array}{l}\text { Not supported Qos, } \\
\text { only to use VLAN }\end{array}$ & fully support 3 layers Qos \\
\hline Configure Method & Manually & Automatically \\
\hline $\begin{array}{l}\text { Automatically Optimize } \\
\text { Audio Delav }\end{array}$ & Not supported & Supported \\
\hline $\begin{array}{l}\text { Same Network Different } \\
\text { Sampling Rate }\end{array}$ & Not supported & Supported \\
\hline $\begin{array}{l}\text { The Master/Slave Network } \\
\text { Redundancy Interface }\end{array}$ & Supported & Supported \\
\hline The Number of Interface & $2 \times 2 / 8 \times 8 / 16 \times 16$ & $8 \times 8 / 64 \times 64 / 128 \times 128$ \\
\hline AVB Update & Not supported & Supported \\
\hline
\end{tabular}

\section{AoIP / AVB Technology Application Prospects in the Broadcasting System}

\subsection{The Change for Transmission Carrier Homogenization}

All along, broadcasting centers are using different types of cables to connect audio devices and network devices, audio dedicated cable balanced microphone cable, digital audio cable, etc., even some stations into audio transmission and computer networks to manage the wiring and system respectively.

Because AoIP / AVB directly using Ethernet / IP network to transmit real-audio signals, as well as having to set up an independent private network like CobraNet, so the audio network and meter machine network can achieve a certain degree integration, its benefits are: Wiring becomes more compact, the system becomes more flexible management more convenient.

At present, almost all mainstream digital mixer with a AoIP interface, the size of interface from $8 \times 8$ to $64 \times 64$ channels ranging, one can put a different mixer output bus master control bundled sent for processing and monitoring, or as a backup master output, on the other hand can be broadcast signal group by the total control over the network directly to the multicast broadcast to live mixer for choosing; All of these with 1 master 1 prepared two cables can fix, in addition to audio signals, equipment operation, room temperature and humidity condition, the video surveillance network data stream and each workstation, can also be included together with the pair of transmission cables. 
Virtual Audio Cable is a great invention, after the installation of Virtual Audio Cable audio in workstation [8], the workstation can record audio directly from AoIP live stream, you can also play audio files sent to the network after the live stream, eliminating AoIPAES3 / Analog - sound - Workstation intermediate links, reducing audio losses, also saves the cost of professional sound card. Virtual Audio Cable supports WDM and ASIO drivers, run all compatible stereo and multitrack production and broadcasting software; Typical applications of this technology is directly used as the network recorded workstation or network slow generation broadcast stations.

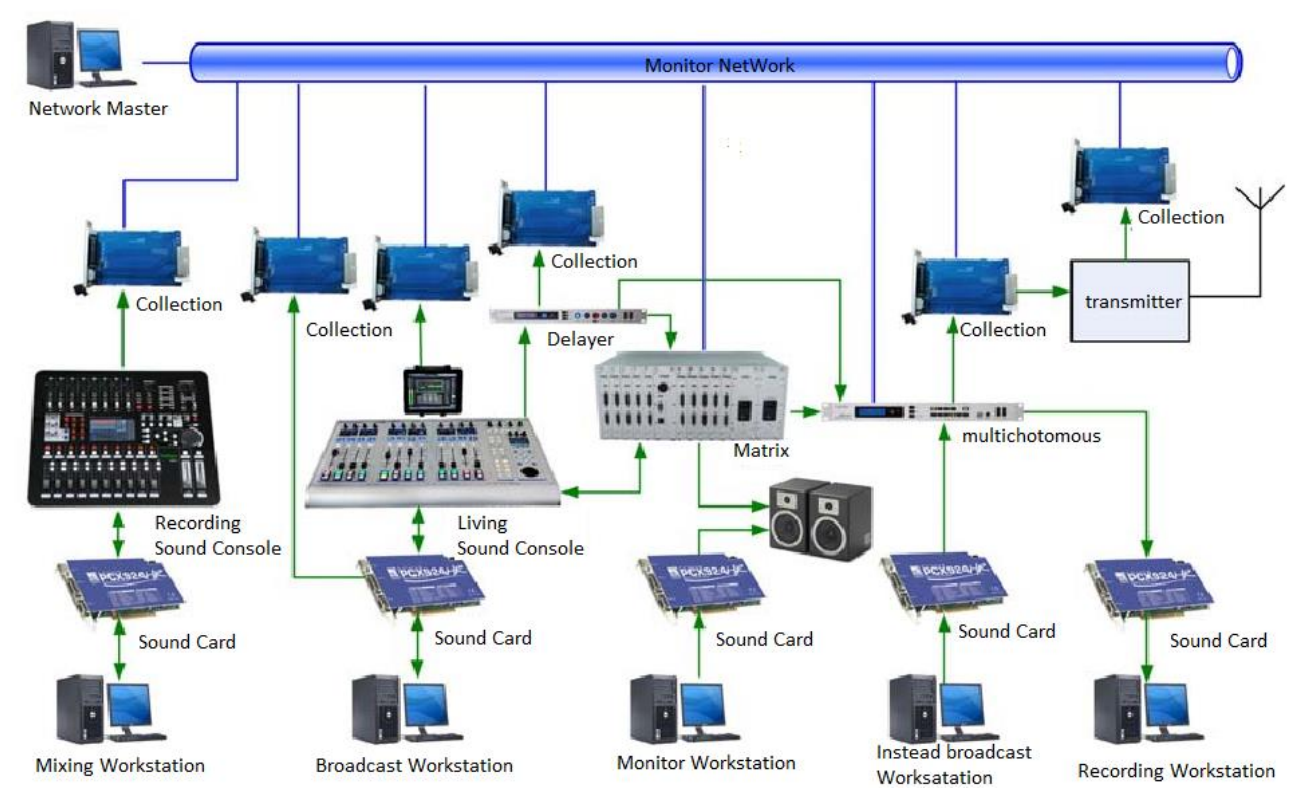

Figure 8. Transmission and Monitoring Systems on Traditional

Some worry interference occurs due to congestion or blocking of data when AoIP networks and computer networks together, in fact, this worry is unnecessary; On the one hand, we are now building a gigabit network have more than one network, all these services together, in fact, is not high relative to the load; On the other hand, we have a QoS mechanism to guarantee priority access time-sensitive data flow, in addition, the main network will limited the risk of Equipment levels and routing level; Of course, about the link signal, we also can do a heterogeneous backup processing with traditional analog or AES3 link. 


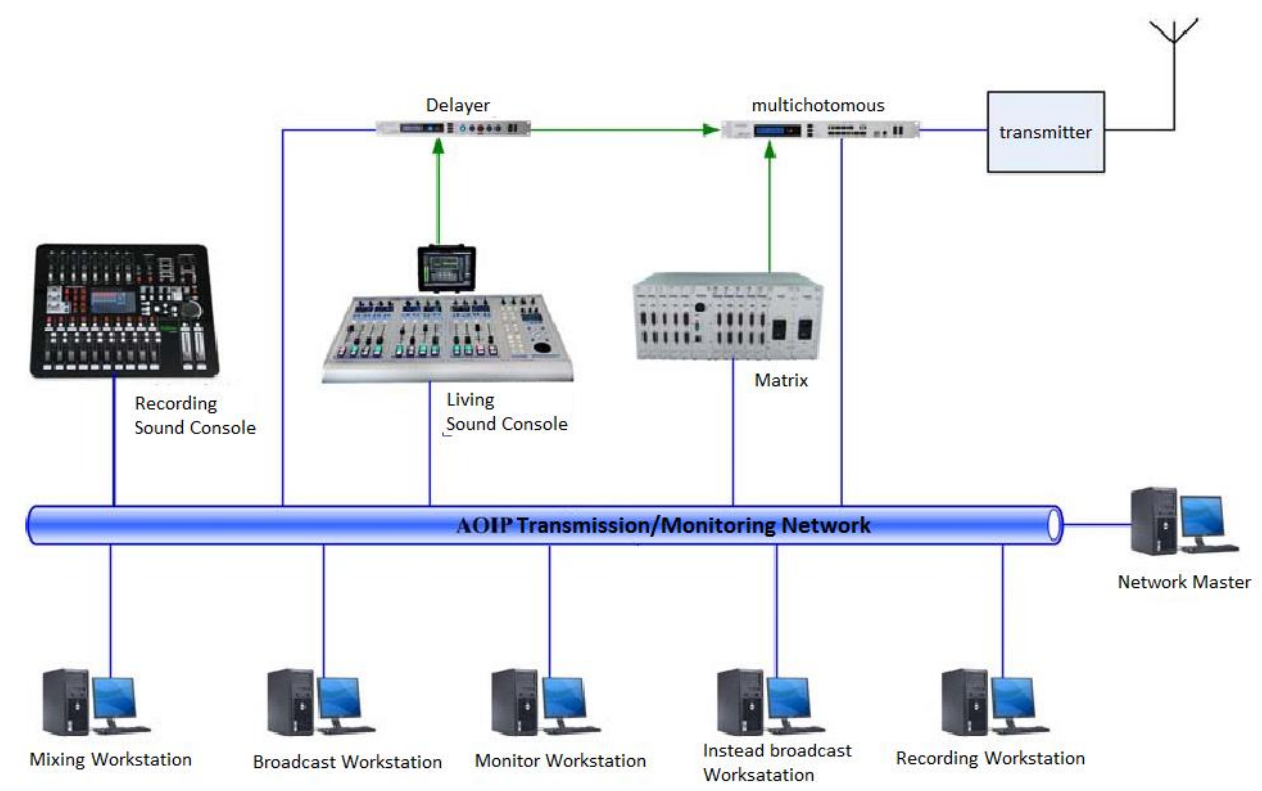

Figure 9. AolP New Generation, Transmission and Monitoring Systems

\subsection{A New Generation of Monitoring and Scheduling Management System}

IP-based devices have easily managed properties, through the LAN, equipment of the entire system may be identified and scheduled by unified network management platform. In previous systems, a lot of traditional device does not have available management interfaces, and they stay out of intelligent systems, even some install network management protocols and interfaces such as SNMP, information can be obtained remains very limited, a large number of audio streams start to be reduced to a level meter again for accident analysis, the reason is not directly sent to the audio data to computing analysis, there must be a pseudo-monitoring risk.

Content-based monitoring is a core feature of the new generation of master control management system, standby signal performed comparative analysis of the content on the same link, get the content relevance, the amount of delay, and noise, frequency response ,clipping, through the relevant algorithm, compared with the previous analysis of the level meter only, it is a qualitative leap; To accomplish this, the audio signal is provided to different nodes brings together, in order to perform in-depth analysis, and this can only rely on AoIP / AVB network can be achieved.

Network and network routing matrix is the product of network audio transmission, can easily change the audio stream to flow through in AoIP network by control protocol, while the use of the multicast function can be easily copying audio stream on transmission node, to achieve networked audio distribution and switching, so we call network matrix. The difference is that the network matrix is dispersed throughout the network without a central device group, not because the failure of individual nodes leads to catastrophic interruption across the board. With the improvement of the control mechanisms, their safety must be much larger than the traditional matrix of the same signal sink cabinet, And at this stage, we often designed to back up each other by conventional matrix and network matrix to strengthen the security of large systems.

AoIP / AVB technology in favor of building a more complete and powerful master control monitoring and management system, mainly in the following aspects:

(1) Device Management: can discovery, configuration and remote management in all network equipment 
(2) Routing management: the traditional routing and network routing analysis unified scheduling, acquisition system link real-time system status, complete backup management and timing switch and emergency switch

(3) Monitoring and Management: Content-based fault detection and alarm management, and complete logging

(4) Monitor management: to monitor the whole network lossless in whole node, a true reflection of the quality of the original

(5) Intercom management: use of network audio transmission technology for remote talkback

(6) Slow recording and generation broadcast: Slow-recorded by a virtual sound card on the specified node, at the same time broadcast signal through the network to the point of failure

\subsection{Achieve The True Digital Audio Cloud Platform}

We are ushering in an era of massive data, also known as the "cloud" era, the development of IT technology making the core network, compute and storage performance becomes more and more powerful; We sampled 24 quantify $48 \mathrm{~K}$ digital audio, for example, its source was $1.152 \mathrm{Mbps} / \mathrm{ch}$, even considering the transmission cost ,overhead in the Fast network environment, the transmission channel can accommodate will reach 56, at gigabit network will exceed 512.If the network is $10 \mathrm{G}, 100 \mathrm{G}$ network, the number will go beyond our traditional imagination; In the broadcast center, media asset library, audio workstations, digital mixer, various sources and broadcast platforms through the same network into one, more and more resources can be shared, more units can be combined and split at any time ,Becoming an all-encompassing, the ubiquitous "Audio cloud."

To terminal, the mixer has no different with workstations absolutely, Because mixer equipped AoIP / AVB function, its input channels and output busses may not is the physical meaning of the input and output, For example, you can start playback via faders on a server, and then packaged into AoIP stream form, and then through the network to participate in the mixing console processing, while bus output can also be turned into a file server directly from AoIP flow; For Audio workstation, you cannot install a sound card, mixing soundtracks can be either real-time track from AoIP device can also be a virtual track from the hard disk. In the audio cloud platforms, no matter the source in where, no matter the signal destination in where, no matter the audio processing unit in where, can be linked together at any time through the network platform, interconnected, mutually substitute, this integrated system resources, enhanced redundancy, reduce system complexity, will be great revolutionary.

\section{Conclusion}

If you use a word to summarize the full text, I would like to use "Yesterday, today and tomorrow" to summarize that "Yesterday -CobraNet, today -AoIP, tomorrow -AVB". The network will bring great potential and facilitate to audio industry, making it into the contemporary huge wave of IT information; Whatever the technology, are deeply imprinted branded with the times, its existence and advantages are relative, cannot simply judge is good or bad, is better; AVB AoIP and development is the same, they are closely related to the transmission and switching technology of network, and directly link with the most advanced MCU and DSP processing power, once its history to open the door, it's advantage will not stop, in the face of tradition transformation will also be the most profound and thorough; We believe that: "Analog - Digital - Network" is the only way for the development of $\mathrm{AV}$, and "higher performance and simpler solution" will be a road brings us huge returns. 


\section{References}

[1] L. Zhu, "IP-based audio conferencing system design", Journal of Xi'an University of Architecture \&Technology, (2010).

[2] M. N. Kevin, "IP audio: The new audio format", Radio Magazine, (2009).

[3] H. B. Yi, "An IP based audio test network for tunnel application", Applied Mechanics and Materials, v (2014), pp. 568-570, pp. 441-447.

[4] J. Xianyang, "An enhanced iot gateway in a broadcast system", Proceedings - IEEE 9th International Conference on Ubiquitous Intelligence and Computing and IEEE 9th International Conference on Autonomic and Trusted Computing, UIC-ATC 2012, (2012), pp. 746-751.

[5] W. Lijuan and X. Baojin, "Talk about Dante Digital Audio Transport Technology", Science and Technology Innovation Herald, no. 2, (2010), pp. 15-16.

[6] AES67-2013, AES standard for audio applications of networks - High-performance streaming audioover-IP interoperability, (2013).

[7] R. Francis, "Audio networking", AES: Journal of the Audio Engineering Society, vol. 60, no. 4, (2012), pp. 282-286.

[8] Y. Rozita, "Analysis of routing protocols of VoIP VPN over MPLS network", Proceedings - 2013 IEEE Conference on Systems, Process and Control, ICSPC 2013, (2013), pp. 139-143.

\section{Authors}

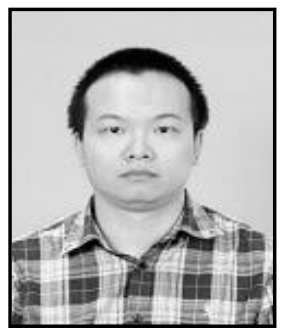

Yi Guo, (1982.11-), Now is working in School of Electrical and Information of Xihua University, and working as University Lecturer. Got the Doctor's degree in June 2013 at School of Automation of University of Electronic Science and technology in the field of Detection technology and automatic equipment. And now major in Pattern recognition and intelligent system, Artificial intelligence, Speech recognition, music transcription, radio and television technology.

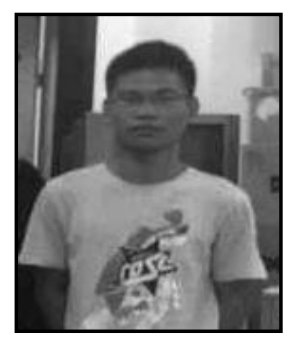

Qiong Li,(1989.10), Now is working in China Electronics Technology Group Corporation No.52 Research Institute in the field of Pattern recognition and intelligent system as an engineer who is in the first year. 
International Journal of Multimedia and Ubiquitous Engineering Vol.11, No.5 (2016) 